\title{
A Critical Study of Coordinated Management of Meaning Theory: A Theory in Practitioners' Hands
}

\author{
Muhammad $\operatorname{Imran}^{1}$, Yuee $\mathrm{CHEN}^{2}$, Xiaofei Matthew WEI ${ }^{1} \&$ Samina Akhtar ${ }^{1}$ \\ ${ }^{1}$ School of Foreign Languages, Shanghai Jiao Tong University, Shanghai, China \\ ${ }^{2}$ School of Foreign Languages, Shanghai University of Finance and Economics, China \\ Correspondence: Muhammad Imran, School of Foreign Languages, Shanghai Jiao Tong University, 800, \\ Dongchuan RD, Minhang District, Shanghai, China. E-mail: imranjoyia76@gmail.com
}

Received: July 10, 2019 Accepted: August 6, 2019 Online Published: August 27, 2019

doi:10.5539/ijel.v9n5p301 URL: https://doi.org/10.5539/ijel.v9n5p301

\begin{abstract}
This paper deals with a critical study of Coordinated Management of Meaning Theory (CMM) as a practical theory and its function to employ a systematic way of conversation in a critical speech. The present study is based on a brief introduction and background perspective of the theory along with its different stages in relation with to address the issues of social conversations. The researcher also has included opinions of many other practitioners of CMM theory and their appreciations, suggestions, and concerns are discussed to make the understanding of the theory in a more clear and comprehensive way. The study concludes that CMM has influenced interpersonal and intercultural communication, management of communication, social communication, conflict, and mediation in communication and last but not the least consultancy and therapy through communication.
\end{abstract}

Keywords: Coordinated Management of Meaning (CMM), conversation, practitioners, communication, consultancy

\section{Introduction}

Barnett Pearce and Vernon Cronen introduced for the first time Coordinated Management of Meaning (CMM) theory in 1978 to address the prevalent social issues and problems to make the social world better. This theory developed from a complex, an interpretive theory to critical theory and finally emerged as a practical theory. The CMM suggests that people's actions form the social worlds around them and the idea behind the development of this theory is that people construct their own social realities and those social realities are formed through dialogues and conversations in which the words and their meaning mean a lot. So, the essence of this CMM theory is participants of any conversation who according to Griffin (2012), "co-construct their own social realities and are simultaneously shaped by the worlds they create" (p. 70).

CMM is an outcome of a debate in the 1970s named 'Great Paradigm Debate' about personal communication issues. The scholars and literary intellectuals took interest in Cronen and Pearce's work and very soon CMM had become popular in literary circles. This theory has decoded the pattern and process of communication and in this way; it allowed the interventions to make the communication better. In this theory, it is suggested that at its initial level that people are reactive and don't passive, however, their behavior can be perceived (Cronen, 2001). Canon and Pearce are of the view that when two people interacts socially, construct the meaning of the conversation they are the part of it. During this conversation, each of the members is also comprised of an interpersonal system which facilitates to explain their action and reaction.

Pearce and Cronen (2001) argue that CMM has much significance in our daily lives. CMM is related to pragmatics due to its aim as what people say and do, and because of constructive nature towards the social world and its interpretation (p. 45). Later in 1967, Peter Berger and Thomas Luckman have presented the ideas in their work 'The Social Construction of Reality' also paved the way for the concept of CMM to be developed in a better way. According to them, reality and knowledge both provide the base for the sociology of knowledge as reality is 'a quality pertaining to phenomena that we recognize as having a being independent of our own volition' and the knowledge, they say 'the certainty that phenomena are real and that they possess specific characteristics' (p. 13). 
CMM represents the views of Mead and Arthur as they reflect that it is a relation of one perspective to the other perspective that is significant as communication is social process and it arises out of cooperative activities (Mead \& Arthur, 1932). John Dewey (1933) has also produced the same views and thoughts about the social process of communication which are later highlighted by CMM, as Dewey says that the problem of practice is what we do need to know, how shall we obtain that knowledge and how shall we apply it. Mead and Dewey both talked about the idea of pragmatics which is inherited by the developers of CMM; Pearce and Cronen.

CMM deals with everyday life conversation of the people among each other and its understanding. According to Griffin (2012), this theory is 'crafted to make life better for real people in the real world' (p. 76). In our daily life, we remain curious about what is going on around us and we try to understand and perceive the world talking to us and around us. Through this understanding we figure out the meaning of the conversation with each other as our understating of the meaning of the words with one person is never be the same with another one. Pearce and Cronen (1980) say that the key idea behind this CMM theory is the creation and deployment of the meaning during conversation is a mutual responsibility of the participants of the conversation (p. 20).

CMM theory has three different directions based on its versatile and complex approach; interpretive, critical and practical. According to Pearce et al. (1988), CMM theory as an interpretive theory deals with our stories; lived and told and those are in tension (p. 30). Griffin (2012) is of the view that CMM theory takes the word management of meaning as referring to the adjustment of the stories narrated in reality to highlight our stories lived and coordinated referring to the constancy of our adjustments while they are interacting with each other (p. 73). Being a critical theory, CMM theory helps to find a channel to involve in communication so that it makes the communication enrich by creating meaning, we coordinate with each other so that a good, desirable and healthy atmosphere can be created instead of bad, ugly and obstructive (Pearce, 2002). Finally, being a practical theory, CMM theory provides a better understanding of the patterns of conversation and interaction to find out the critical moments in conversation as Griffin (2012) suggests that it assists to find ways to talk that will create a better social environment (p. 67). In other words, it is a theory which can be put into practice easily. The founders of this theory, Pearce and Cronen, believe that this theory offers practical tools that include teachers, therapists, consultants, social workers, and mediators to create a healthy social environment. In brief, according to Pearce and Cronen (1980), the basic aim of this CMM theory is to discuss the human condition (p. 177). This human condition is our own production in a collective way as both created and managed by us to produce a social reality so this aim helps to understand the term Coordinated Management of Meaning (CMM) in an easy way to comprehend it.

\subsection{Components of CMM Theory}

The term has been divided into three parts; coordinated, management and meaning. Pearce and Cronen (1980) are of the view that coordinated implies interpersonal rule systems, the management is, of course, the masses, the persons involved in the conversation and the meaning is a stakeholder of the form of communication (p. 123). Every part of the theory is vital in its nature and integral role to the various facets of CMM theory. CMM theory is closely related to M. C. Escher's 'Bond of Union' as Griffin (2012) has quoted it in his 'A First Look at Communication Theory'. According to 'Bond of Union' we all are interrelated to each other in one or the other way. With the passage of time, people become more critical in thinking and interaction and try to choose what suit them to take it for a long time with them. Nobody lives and wants to live in an isolated life as we need people around us to make and share ideas, feelings, and stories. This critical process covers the whole life of the man from childhood to old age. In childhood, a person depends more upon parents for communication and interaction and as he/she grows older, become more critical and logical in thinking and interactions.

Through interaction and conversation with people, the communication occurs and CMM helps to create social realities in daily life by suggesting and assigning meaning to each piece of the conversation. The construction of the meaning is very significant and critical as well. The word 'meaning' is very broad in its literal sense. The founders of CMM theory, Pearce and Cronen (1980) have described the word 'meaning' in three different ways. They are of the view that the 'meaning'; 1) the meaning is not taken from any experience but it is an imposed entity on the name of experience, 2) the meaning has required a comparable framing for coordinated communication to occur and 3) for the assumption and perception of meaning, coordinated communication is observed to occur across centuries and cultures (p. 138). From these assumptions about the word 'meaning', it has become clear that people compare the world through their own understanding. Their level of assumption about my any word is based upon their level of knowledge and awareness about the world around them. Pearce and Cronen (1980) further relate the meaning and its understanding during a conversation with speech acts (p. 132). These speech acts can be any kind of assumption based on the speakers' or learners' level of understanding such as those speech acts can be a promise, insult, complaint, threat or any other kind of meaning the people 
have assigned to it.

\section{The CMM Theory in Practitioners' Hands}

This section of the article intends to designate the attention towards the views of those who engaged themselves to use CMM theory practically or theoretically and made criticism on it. Griffin (2012) in his book A First Look at Communication Theory, argues that Pearce and Cronen (1980), the founders of CMM theory, believe that our collective participation in social events and objects of the world around makes the process of the communication successful. CMM theory is based on the concept that 'persons-in-conversation co-construct their own social realities and are simultaneously shaped by the worlds they create'. This notion leads towards the basic idea of CMM as Pearce and Cronen (1980) lay stress on 'the things we do' to each other and 'the things we make' with each other while interaction and conversation. Griffin has evaluated this theory on three parameters: thoroughness, originality, and accuracy. He has discussed the details of the theory in his introductory pages of analysis along with the origin of the CMM theory and its historical aspects. His focus is more concerned with the understanding of the concept of the CMM theory by being interpretive, practical and critical. According to him, the practical application of CMM theory can be perceived by three different situations; a family therapist's case, a mediator's couple case and the Cupertino project to highlight that to what extent CMM theory is treated as a practical theory. By interpretive, he describes the characteristics of CMM theory through a different example to make it more understandable such as the Bond of Union lithograph to illustrate the notion of persons in conversation. At last, he throws light on the critical application of this CMM theory as it is a way to intervene the logical force of dialogues and conversations to make it different from the useless and unhealthy discourse and communication. Griffin has also quoted the example of Martian Buber's dialogic parameters which included ethics and findings, case studies from the original text of Pearce and Cronen along with stories and expert opinions. One thing significant in Griffin's analysis of CMM theory is that he couldn't be able to define the tools for the methodology of the CMM theory working and in case of any alteration in the defined system.

By originality in CMM theory, Griffin (2012) means the examples from real-life situations. He has described Calvin and Hobbes comic strip to point out the constructs, coherence without coordination and the mixing of the real-life stories which are still alive (p. 76). One prominent example is Ex-US President George W. Bush's speech on the dreadful mishap of 9/11 night and he labeled these as 'war of terror'. According to Pearce, in his speech, the president has had the opportunity to resolve the matter in a better way through his use and selection of words during his conversation about that particular incident. And with the concept of accuracy, Griffin thinks that CMM theory is reliable in its application in real-life situations. He himself in his analysis of CMM theory has used clear and simple examples form real-life situation to show the accuracy of the theory.

West and Turner (2000) in their book Introducing Communication Theory: Analysis and Application have discussed in detail the CMM theory by mentioning its strengths and considering points pointed by other critics. CMM theory has placed communication as a basic counter stone because communication is the central mark of it. Many literary icons have used CMM theory in scholarly writings as researchers have used this theory to resolve the conflicts and understand those tents of the conflicts (Pearce \& Littlejohn, 1997), cultural communities (Narula \& Pearce, 1986), conversations (Pearce \& Conklins, 1979), groups (Pearce \& Pearce, 2000), families (Cronen et al., 1982), organizations (Cronen et al., 1979) and public address (Branham \& Pearce, 1985). According to the practitioners of the CMM theory, this theory is useful as it is practical and very broad in its scope because it deals with both intrapersonal and interpersonal processes of communication. On the other hand, some critics have considered a few areas despite the strengths of CMM theory. David Brenders (1987) points out that as Pearce and Cronen (1980) claim that individuals have a unique role in producing language while interacting with others as for CMM theory meanings are an intrapersonal experience. Here Brenders (1987) counters the argument and says that 'we share a language which is not our idiosyncratic production, but a medium of shared symbolic meanings' (p. 342).

Anderson (1996) is of the view that CMM theory has provided us the way by which we can look at how individuals co-create their meaning in deeper understanding. He pays thanks to CMM theory for sharing a large part in the struggle to understand the dynamic interplay of interpersonal and intrapersonal communication. CMM theory has further helped us to make more understanding about the rules and their importance as to how to deal with real social situations with conversation and interaction. It seems CMM theory has assigned the central place to communication for human interaction experiences. This contribution has made the CMM theory among one of the few communication theories that are 'firmly in the world it describes' (p. 209).

Wood (1997) in Communication Theories in Action: An Introduction has discussed CMM theory from three perspectives which are considered the focal issues in CMM theory analysis by the critic. She has addressed the 
issues of: 1) unclear meaning of rule as Susan Shimanoff (1985) describes that rules are based on observable behavior particularly because it's not necessary that all rules theorists accept the meaning rule of basic ideas while CMM theory founders Pearce and Cronen (1980) argue that the rules appertain to internal, subjective interpretation as well as to overt activities. 2) Too ambiguous nature of CMM theory is also criticized as the rule theories always have been criticized for their unclarity and ambiguity. The definition of the word 'rule' is controversial and no agreement is made on one definite definition. This ambiguity goes toward the question that whether rules theory deals well with unexpected forms of communication. The rule theorists don't have made the precise and to the point rules of the violation, this is the reason creative and innovative behaviors find it difficult to analyze the framework of this theory. 3) Too broad in scope, CMM theory is mostly criticized due to its broadness in scope and even the supporters of CMM theory have the same complaint against this theory. According to Brenders (1987), the CMM theory tries to elaborate the whole universe from different problems faced between cultures to difficulties in close relationships. In this way, critics are of the view that this CMM theory has become fail to adopt the comprehensiveness and adaptability as it lacks the necessary precision to allow peeping through the specific communication segments and activities.

Hall (1992) in his article titled as Theories of Culture and Communication makes a comparative study of culture and communication and in a third-round as the relationship between the two: culture and communication as the perspectives of traditional, coordinated management of meaning and ethnography of communication.

Rose (2006) in the essay A Proposal for Integrating Structuration Theory with Coordinated Management of Meaning Theory shows how the CMM theory and structuration theory are inter-related to each other. The whole essay deals with the discussion based on the aspects of these two theories and suggest that the narrative meaning frameworks of CMM theory are closely linked with the modality of structuration theory. It is articulated that this deal between two theories opens up many connections in terms of the development of positioning, level of interaction and institutional analysis.

Buttle (1994) in 'The Coordinated Management of Meaning: A Case Exemplar of a New Consumer Research Technology' published in European Journal of Marketing says that CMM theory is a theory based on human actions and reactions which make it a social constructionist theory. It helps in making people aware and taking decisions in a situation of multiple involvements of the people in one matter so that they can take any collective decision in a better way. He has seen this CMM theory in a very unique way as it is helpful in consumer and marketing paradigm and he gives a real-life reference from the story of the Hughes Family' decision about to spend the holidays and decision about the supply of necessary items. In such case, CMM theory supplies an episode within which the family's socially constructed resources are described and made happy for them.

Salmon and Faris (2006) in their essay 'Multi-agency collaboration, multi-levels of meaning: social constructionism and the CMM model as tools to further understanding appeared in Journal of Family Therapy. They have discussed in detail CMM theory and its usefulness as it is helpful in understanding and analysis of the data for multi-agency meetings. They have given value to thematic analysis as it gives the study of nine themes and becomes beneficial to be defined with reference to discourse type, agency, case complexity, and multi-agency, etc.

Bruss et al. (2005) in Food, Culture and Family: Exploring the Coordinated Management of Meaning Regarding Childhood Obesity argue that the child health obesity needs proper address to be solved and spread awareness among children and parents so that the information related to physique, diet, growth and chronic diseases based on the information about child feeing practices. For this purpose, the considerations of individuals and collectives based on familial, cultural, social and official nutritional discourses are very significant to improve the behaviors about health care issues. The CMM theory is found helpful in interpreting the messages conveyed to the public in negotiating the content and context occurred at different levels to conceive the meanings. According to them, the CMM theory proved to be very close in understanding the beliefs and values about the feeding of the children along with the attitude and practices of the guardians in different groups of the population. Implications for future health communication research that might draw from a CMM theory approach were identified as well as pragmatic endeavors that focus on the development, implementation, and evaluation of culturally appropriated interventions in the prevention of childhood obesity.

Oliver and Brittain (2000) in Systemic Constructionist Management elaborated that how CMM theory has multiple influences on management and organization. They have sorted out that it has a strong influence in management training organization as well. There were confusion and tension about the definitions, visions, and meanings of management within the organization but Oliver and Brittain proposed the solution with CMM theory as to see the complexity and of the organization. With the help of CMM theory, they became able to 
introduce their domains model to construct a frame for a talk in which they have placed different alternatives such as appreciation instead of frustration, leading instead of hankering and coaching the organization through the proposed model. By using the CMM theory patterns and elements, the trainers and practitioners were given space to apply and think for different patterns of communication within a specific organization.

\section{A Personal Note on CMM as Practical Theory}

According to Cronen's (1980) explanation and general assessment for CMM theory as practical theory, he has suggested some standards for practical theory in the following: 1) practical theory focuses on the representation of real-world actions, 2) practical theory suggests its practitioners be flexible in sharing and joining the principles of others' grammars, 3) practical theory looks forward to the betterment of social lives, 4) practical theory should be persistent, and defensible internally and 5) practical theory has always a reliable and flexible relation with grammars in action to influence the practical theory in any form and shape. By measuring these principle standards as for a practical theory, CMM theory well deserves to be a practical theory as it has owned many tools which include hierarchy of meaning, and strange loops to develop coordinated patterns within human systems to produce a better social environment in human condition can be made better and improved (Cronen \& Pearce, 1980; Cronen, Johnson, \& Lannmann, 1982). There are certain issues regarding ethics, reflexivity and utility have been addressed here which are sorted out by the practitioners of CMM theory as practical theory. Those who are practitioners of CMM theory should need to know that why they are engaged with this theory so that they may better know the conditions and ethics in which they can use the CMM theory in a better way. The practitioners should take a communication perspective about the practical approach of CMM theory as it helps to make perspectives of communication in a specific phenomenon such as leadership, intercultural communication, and mediation.

\section{Conclusion}

It is observed that CMM theory plays an important and vital role as a practical theory in better understanding of the meaning and daily conversations. It is very significant in making the social world better as proved by the discussions of various practitioners of this theory in practical fields have been appreciated. This theory developed from a complex, an interpretive theory to critical theory and finally emerged as a practical theory. The CMM suggests that people's actions form the social worlds around them and the idea behind the development of this theory is that people construct their own social realities and those social realities are formed through dialogues and conversations in which the words and their meaning means a lot. This theory is pregnant with plenty innovations and rapidly growing needs of communication among different members of society forecast that with the three development stages of CMM as interpretive, critical and practical theory there must be a fourth one, I don't know what it will be but it seems through its growth that there must astonishing development in the near future.

\section{References}

Anderson, R. J. (1996). ACT: A simple theory of complex cognition. American Psychologist, 51, 355-365. https://doi.org/10.1037//0003-066X.51.4.355

Berger, L. P., \& Luckman, T. (1967). The Social Construction of Reality: A Treatise in the Sociology of Knowledge. New York: Anchor.

Bruss, J., Dennison, O., Quitugua, P., \& Palacios, T. (eds). (2005). Food, Culture, and Family: Exploring the Coordinated Management of Meaning Regarding Childhood Obesity. Health Communication, 18, 155-175. https://doi.org/10.1207/s15327027hc1802_4

Buttle, F. (1994). The Coordinated Management of Meaning: A Case Exemplar of a New Consumer Research Technology. European Journal of Marketing, 28(8/9), 76-99. https://doi.org/10.1108/03090569410067640

Cronen, V. (2001). Practical theory, practical art, and the pragmatic-systemic approach to inquiry. Communication Theory, 11, 14-35. https://doi.org/10.1111/j.1468-2885.2001.tb00231.x

Cronen, V., \& Pearce, B. (eds.) (1988). Coordinated management of meaning: A critical theory. International and Intercultural Communication Annual, 12, 66-98.

Dewey, J. (1938). Logic: The theory of inquiry. New York: Basic Books.

Griffin, E. (2012). A First Look at Communication Theory (8th ed.). Boston, MA: McGraw-Hill.

Hall, B. (1992). Theories of culture and communication. Communication Theory, 2, 50-71. https://doi.org/10.1111/j.1468-2885.1992.tb00028.x 
Mead, A. (1932). The Philosophy of the Present. Chicago: Open Court.

Oliver, C. (2000). Systemic Eloquence. Human Systems: The Journal of Systemic Consultation and Management, $7(4)$.

Pearce, K. (2002). Making better social worlds: Engaging in and facilitating dialogic communication. Pearce Associates.

Pearce, W. B., \& Cronen, V. E. (1980). Communication, action, and meaning: The creation of social realities. New York: Praeger.

Rose, R. (2006). A proposal for integrating structuration theory with coordinated management of meaning theory. Communication Studies, 57, 173-196. https://doi.org/10.1080/10510970600666867

Salmon, G., \& Faris, J. (2006). Multi-agency collaboration, multiple levels of meaning: Social constructionism and the CMM model as tools to further our understanding. Journal of Family Therapy, 28(3), 272-292. https://doi.org/10.1111/j.1467-6427.2006.00352.x

West, R., \& Turner, L. (2000). Introducing communication theory: Analysis and application. Mountain View, CA: Mayfield.

Wood, J. (1997). Communication Theories in Action: An Introduction. Belmont, CA: Wadsworth.

\section{Copyrights}

Copyright for this article is retained by the author, with first publication rights granted to the journal.

This is an open-access article distributed under the terms and conditions of the Creative Commons Attribution license (http://creativecommons.org/licenses/by/4.0/). 